\title{
An oscillatory bifurcation from infinity, and from zero
}

\author{
Philip Korman
}

\begin{abstract}
The problem (where $B$ is a unit ball in $R^{n}$ )
$\Delta u+\lambda(u+u g(u))=0, \quad x \in B, \quad u=0$ for $x \in \partial B$,

with $\lim _{u \rightarrow \infty} g(u)=0$, is known to have a curve of positive solutions bifurcating from infinity at $\lambda=\lambda_{1}$, the principal eigenvalue. It turns out that a similar situation may occur, when $g(u)$ is oscillatory for large $u$, instead of being small. In case $n=1$, we can also prove existence of infinitely many solutions at $\lambda=\lambda_{1}$ on this curve. Similarly, we consider oscillatory bifurcation from zero.
\end{abstract}

Mathematics Subject Classification (2000). 34B15, 35J60.

Keywords: Bifurcation from zero, and from infinity, infinitely many solutions.

\section{Introduction}

If one considers the problem

$$
u^{\prime \prime}+\lambda a(x)(u+g(u))=0,-1<x<1, u(-1)=u(1)=0,
$$

with $\lim _{u \rightarrow \infty} \frac{g(u)}{u}=0$, then there is a curve of positive solutions bifurcating from infinity at $\lambda=\lambda_{1}$. (Let us recall that $\lambda_{1}=\frac{\pi^{2}}{4}$, and $\phi_{1}(x)=\cos \frac{\pi}{2} x$ form a principal Dirichlet eigenpair of $-u^{\prime \prime}$ on the interval $(-1,1)$.) Intuitively, for large positive solutions the $g(u)$ term is negligible, and hence $\lambda$ must be close to $\lambda_{1}$, while $u(x)$ is then close to a multiple of $\phi_{1}(x)$. It turns out that a completely similar situation may occur, when $g(u)$ is oscillatory for large $u$, instead of being small. This appears to be a new phenomenon, which we refer to as oscillatory bifurcation, in contrast with the well studied bifurcation from infinity case. Similarly, we discuss oscillatory bifurcation from zero. We obtain the most detailed results if the problem (1.1) above is either autonomous, or $a(x)$ is an even function, with $a^{\prime}(x) \leq$ 0 for $x>0$. Namely, we show existence of infinitely many positive solutions at $\lambda=\lambda_{1}$ for both the cases of oscillatory bifurcation from infinity and from zero. 
The proofs are based on a lemma from complex analysis, which we review in the next section. Our approach is similar to that of A. Galstyan et al [5]. Our results are confirmed by numerical computations, one of which we present below.

We describe next our approach to the oscillatory bifurcation from infinity. For the problem (1.1) we begin with a curve of positive solutions, which bifurcates from zero (according to the standard results of M.G. Crandall and P.H. Rabinowitz [3]), and then show that this curve tends to $\left(\lambda_{1}, \infty\right)$ in an oscillatory way. It would be desirable to develop a priori existence results near $\left(\lambda_{1}, \infty\right)$, similarly to the standard results on bifurcation from infinity.

We derive similar results also for a more general class of non-autonomous problems, and for balls in $R^{n}$. In both of these cases we use a different technique to study the solution curve near infinity, which is similar to R. Schaaf and K. Schmitt [13]. The basic approach remains the same. We begin with a curve of positive solutions, bifurcating from zero, and show that the oscillatory nature of the nonlinear term causes this curve to go to infinity at $\lambda=\lambda_{1}$.

\section{An oscillatory bifurcation from infinity: a model example}

We recall an elementary lemma from complex analysis, which shall be our principal tool in this section. It is a small modification of the corresponding result in Y.V. Sidorov et al [12], see also [5], where the functions $f$ and $g$ were assumed to be independent of $\mu$.

Lemma 2.1. Let $\mu$ be a positive parameter, and consider the functions $f(x, \mu) \in$ $C^{2,0}\left([-1,1] \times R_{+}\right)$and $g(x, \mu) \in C^{2,0}\left((-1,1) \times R_{+}\right)$. Assume that for all $\mu$, $x=0$ is the only stationary point of $g(x, \mu)$ on $[-1,1]$, i.e $\left(g_{x}(0, \mu)=0\right)$, and $g_{x x}(0, \mu) \neq 0$. Then as $\mu \rightarrow \infty$

$$
\int_{-1}^{1} f(x, \mu) e^{i \mu g(x, \mu)} d x=e^{i\left(\mu g(0, \mu)-\frac{\pi}{4}\right)} \sqrt{\frac{\pi}{\mu\left|g_{x x}(0, \mu)\right|}} f(0, \mu)+O\left(\frac{1}{\mu}\right) .
$$

Corollary 1. In particular, the lemma implies that the integral on the left tends to zero as $\mu \rightarrow \infty$, provided that $f(0, \mu)$ is bounded for all $\mu$.

We consider positive solutions of a model problem

$$
u^{\prime \prime}+\lambda\left(u+\frac{1}{2} u \sin u\right)=0,-1<x<1, u(-1)=u(1)=0,
$$

depending on a positive parameter $\lambda$. It is known that all positive solutions of (2.1) are even functions, with $x u^{\prime}(x)<0$ for $x \neq 0$, and they lie on a smooth solution curve, see e.g. P. Korman [8]. The following lemma shows that the solution curve of (2.1) lies in a strip of $(\lambda, u(0))$ plane. 
Lemma 2.2. If the problem (2.1) has a positive solution, then

$$
\frac{2}{3} \lambda_{1}<\lambda<2 \lambda_{1} \text {. }
$$

Proof. Multiplying the equation (2.1) by $u$, and integrating

$$
\frac{3}{2} \lambda \int_{-1}^{1} u^{2} d x>\lambda \int_{-1}^{1}\left(u^{2}+\frac{1}{2} u^{2} \sin u\right) d x=-\int_{-1}^{1} u u^{\prime \prime} d x \geq \lambda_{1} \int_{-1}^{1} u^{2} d x,
$$

from which the first inequality in (2.2) follows. If the second inequality in (2.2) was false, then we would have $g(u) \equiv \lambda\left(u+\frac{1}{2} u \sin u\right)-\lambda_{1} u=\left(\frac{\lambda}{2}-\lambda_{1}\right) u+$ $\lambda\left(\frac{1}{2} u+\frac{1}{2} u \sin u\right)>0$ for almost all $u>0$. However, multiplying the equation (2.1) by $\phi_{1}$, and integrating

$$
\int_{-1}^{1} g(u) \phi_{1} d x=0
$$

which is a contradiction.

Theorem 2.1. There is a curve of positive solutions of the problem (2.1), bifurcating from zero at $\lambda=\lambda_{1}$. This curve intersects the line $\lambda=\lambda_{1}$ infinitely many times, and it tends to infinity as $\lambda \rightarrow \lambda_{1}$. Moreover, as this curve tends to infinity, the solution tends to a constant multiple of $\phi_{1}(x)$.

Proof. By the standard theory of bifurcation from zero, there is a curve of positive solutions bifurcating (backward in $\lambda$ ) from zero at $\lambda=\lambda_{1}$, see [3]. This branch continues globally, since at each point either the implicit function theorem or bifurcation theorem of M.G. Crandall and P.H. Rabinowitz [4] applies, see e.g. [8] for details. By Lemma 2.2 the solution curve is constrained to a strip $\frac{2}{3} \lambda_{1}<\lambda<$ $2 \lambda_{1}$, and hence it goes to infinity inside this strip.

We show next that the solution curve is asymptotic to the line $\lambda=\lambda_{1}$, and it crosses this line infinitely many times. We let $u=\mu v$ in (2.1), with $\mu=u(0)$, the maximum value of the solution, and $v(0)=1$. Then (2.1) transforms to

$$
v^{\prime \prime}+\lambda\left(v+\frac{1}{2} v \sin \mu v\right)=0,-1<x<1, \quad v(-1)=v(1)=0 .
$$

It is known that $\mu$ is a global parameter on the solution curve, i.e., $v=v(x, \mu)$ and $\lambda=\lambda(\mu)$, see e.g. [8]. For simplicity, we shall suppress the dependence on $\mu$, and write $v=v(x)$. Multiplying the equation (2.3) by $\phi_{1}$, and integrating yields

$$
\left(\lambda_{1}-\lambda\right) \int_{-1}^{1} v(x) \phi_{1}(x) d x=\frac{1}{2} \lambda \int_{-1}^{1} v(x) \sin \mu v(x) \phi_{1}(x) d x .
$$


We observe that the integral on the right can be written as an imaginary part of a complex integral $\Im \int_{-1}^{1} v(x) \phi_{1}(x) e^{i \mu v(x)} d x$, to which we apply the Lemma 2.1 .

$$
\int_{-1}^{1} v(x) \phi_{1}(x) e^{i \mu v(x)} d x=e^{i\left(\mu-\frac{\pi}{4}\right)} \sqrt{\frac{\pi}{\mu\left|v^{\prime \prime}(0)\right|}}+O\left(\frac{1}{\mu}\right) .
$$

Using this in (2.4), we have

$$
\left(\lambda_{1}-\lambda\right) \int_{-1}^{1} v(x) \phi_{1}(x) d x=\frac{1}{2} \lambda \sin \left(\mu-\frac{\pi}{4}\right) \sqrt{\frac{\pi}{\mu\left|v^{\prime \prime}(0)\right|}}+O\left(\frac{1}{\mu}\right) .
$$

From the equation (2.3) and Lemma 2.2, we conclude that

$$
\frac{1}{3} \lambda_{1}<\frac{1}{2} \lambda \leq\left|v^{\prime \prime}(0)\right| \leq \frac{3}{2} \lambda<3 \lambda_{1}
$$

We see from the equation (2.3) that the function $v(x)$ is concave, and since $v(0)=1$, it follows that $v(x)>1-|x|>0$, and hence the integral $\int_{-1}^{1} v(x) \phi_{1}(x) d x$ is bounded from below by a positive constant. We then conclude from (2.5) that $\lambda_{1}-\lambda$ changes sign infinitely many times, and it tends to zero, as $\mu \rightarrow \infty$.

Turning to the last claim, we rewrite the problem (2.3) as

$$
v^{\prime \prime}+\lambda_{1} v=\left(\lambda_{1}-\lambda\right) v+\frac{\lambda}{2} v \sin \mu v,-1<x<1, \quad v(-1)=v(1)=0 .
$$

Using the modified Green's function $G(x, \xi)$, see e.g. p. 136 in E.C. Young [15], we express

$$
v(x)=\left(\lambda_{1}-\lambda\right) \int_{-1}^{1} G(x, \xi) v(\xi) d \xi+\frac{\lambda}{2} \int_{-1}^{1} G(x, \xi) v(\xi) \sin \mu v(\xi) d \xi+c \phi_{1}(x),
$$

where $c$ is some constant. The first term on the right tends to zero, since $\lambda \rightarrow \lambda_{1}$, as $\mu \rightarrow \infty$, while the second one tends to zero by applying the Corollary 1 , in a way similar to what we just did. It follows that $v(x)$ tends to $c \phi_{1}(x)$.

\section{An oscillatory bifurcation from zero: a model example}

We consider positive solutions (of class $C[0,1] \cap C^{2}(0,1)$ ) of a model problem

$$
u^{\prime \prime}+\lambda\left(u+\frac{1}{2} u \sin \frac{1}{u}\right)=0,-1<x<1, u(-1)=u(1)=0,
$$

depending on a positive parameter $\lambda$. The classical result of M.G. Crandall and P.H. Rabinowitz [3] on bifurcation from zero does not apply here. However, we 
show that bifurcation from zero at $\lambda_{1}$ does indeed happen, and the solution curve makes infinitely many turns in any neighborhood of $\left(\lambda_{1}, 0\right)$, see Figure 1 for the bifurcation diagram computed by Mathematica (for the problem posed on the interval $x \in(0,1))$. For the description of the numerical method we used, please see our review paper [8].

Theorem 3.1. There is a curve of positive solutions of the problem (3.1), bifurcating from infinity at $\lambda=\lambda_{1}$. This curve tends to zero as $\lambda \rightarrow \lambda_{1}$. Moreover, in any neighborhood of zero, this curve intersects the line $\lambda=\lambda_{1}$ infinitely many times, and the solution tends to a constant multiple of $\phi_{1}(x)$.

Proof. The proof is similar that of Theorem 2.1, so we shall be brief. By standard results on bifurcation from infinity, there is a curve of positive solutions, bifurcating from infinity at $\lambda=\lambda_{1}$. Similarly to Lemma 2.1, we see that positive solutions of (3.1) are constrained to a strip

$$
\frac{2}{3} \lambda_{1}<\lambda<2 \lambda_{1} .
$$

As before, we let $u=\alpha v$ in (3.1), with $\alpha=u(0)$, and $v(0)=1$. Then (3.1) transforms to

$$
v^{\prime \prime}+\lambda\left(v+\frac{1}{2} v \sin \mu \frac{1}{v}\right)=0,-1<x<1, \quad v(-1)=v(1)=0,
$$

where $\mu=1 / \alpha$. Observe that $\mu \rightarrow \infty$, as $\alpha \rightarrow 0$ (i.e. when $u$ tends to zero). Multiplying the equation (3.2) by $\phi_{1}$, and integrating

$$
\left(\lambda_{1}-\lambda\right) \int_{-1}^{1} v(x) \phi_{1}(x) d x=\frac{1}{2} \lambda \int_{-1}^{1} v(x) \sin \mu \frac{1}{v(x)} \phi_{1}(x) d x .
$$

Again, we observe that the integral on the right can be written as an imaginary part of a complex integral $\Im \int_{-1}^{1} v(x) \phi_{1}(x) e^{i \mu \frac{1}{v(x)}} d x$, to which we apply the Lemma 2.1 (observe that the second derivative of $\frac{1}{v(x)}$ at zero is $-v^{\prime \prime}(0)$ )

$$
\int_{-1}^{1} v(x) \phi_{1}(x) e^{i \mu \frac{1}{v(x)}} d x=e^{i\left(\mu-\frac{\pi}{4}\right)} \sqrt{\frac{\pi}{\mu\left|v^{\prime \prime}(0)\right|}}+O\left(\frac{1}{\mu}\right) .
$$

As before, we conclude from (3.3) that $\lambda_{1}-\lambda$ changes sign infinitely many times, and it tends to zero, as $\mu \rightarrow \infty$. (As before, $\int_{-1}^{1} v(x) \phi_{1}(x) d x$ does not tend to zero, since the concave function $v(x)$ satisfies $v(0)=1$.) 


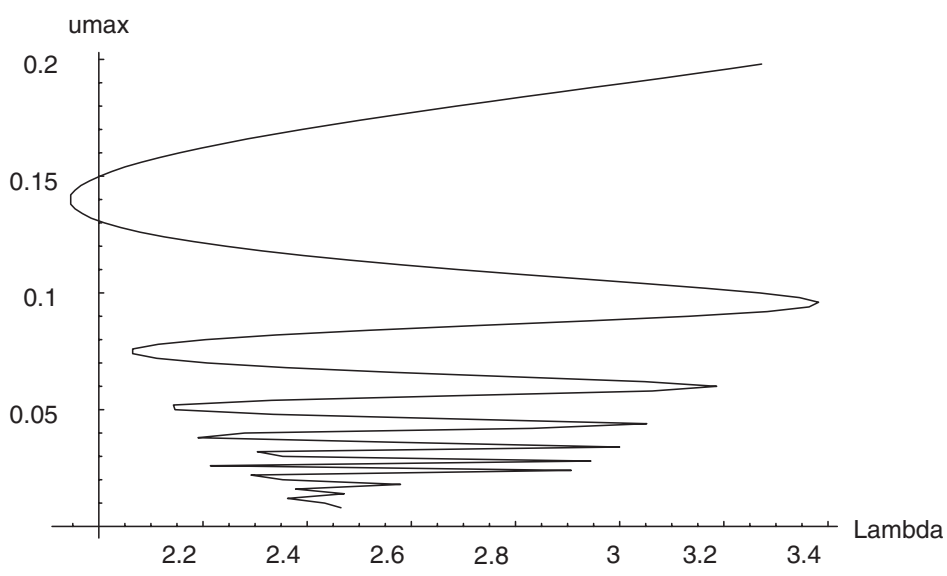

Figure 1 Bifurcation diagram near zero for the problem (3.1)

\section{More general results}

Clearly our results are not limited to the above examples. For example, the Theorem 2.1 will hold verbatim for boundary value problems of the type

$$
u^{\prime \prime}+\lambda a(x)(u+\alpha u \sin u)=0, \quad-1<x<1, \quad u(-1)=u(1)=0 .
$$

Here we assume that $\alpha \neq 0$ is a constant, with $|\alpha|<1$ (to make sure the nonlinear term is non-negative). We assume $a(x) \in C^{1}(-1,1) \cap C[-1,1]$ to be positive on $[-1,1]$, even, and $x a^{\prime}(x) \leq 0$ for $x \neq 0$. For such $a(x)$ the problem (4.1) has properties similar to those of autonomous problems, see [10] or [8] (any positive solution $u(x)$ is even, unimodular, with maximum at $x=0$, and all positive solutions of (4.1) lie on one smooth curve). In particular, if $\alpha=-\frac{1}{2}$, we have the problem

$$
u^{\prime \prime}+\lambda a(x)\left(u-\frac{1}{2} u \sin u\right)=0,-1<x<1, u(-1)=u(1)=0,
$$

thus generalizing one of the results in a recent paper of S.-H. Wang [14]. Similarly, we can generalize the Theorem 3.1.

Next, we shall derive a similar result in a considerably more general set up, with variable coefficients. Since we do not assume any symmetry, we pose the problem on the interval $(0,1)$ :

$$
\begin{aligned}
u^{\prime \prime}+\lambda a(x)(u+u g(u)) & =0, \text { for } 0<x<1, \\
u(0)=u(1) & =0 .
\end{aligned}
$$

Here $a(x) \in C^{1}[0,1]$ is a given functions, with $a(x)>0$ on $[0,1]$, and in addition 
one of the following conditions is assumed to hold: either

$$
\frac{3}{2} \frac{a^{\prime}(x)^{2}}{a(x)}-a^{\prime \prime}(x)<0 \text { for all } x \in(0,1),
$$

or

$$
a^{\prime}(x)<0 \text { and } x a^{\prime}(x)+2 a(x)>0 \text { for all } x \in(0,1) .
$$

For the nonlinear term $g(u) \in C^{1}\left(\bar{R}_{+}\right)$we assume that

$$
g(0)=0 \text {, and }|g(u)| \leq g_{0}<1 \text { for all } u>0 \text {, and some constant } g_{0} .
$$

For any $u>0$ we define the functions $G(u)=\int_{0}^{u} g(t) d t$, and $H(u)=\int_{0}^{u} G(t) d t$. We assume that

$$
\frac{1}{\mu} G(\mu v) \text { and } \frac{1}{\mu^{2}} H(\mu v) \text { tend to } 0 \text {, as } \mu \rightarrow \infty \text {, uniformly in } v \in[0,1] .
$$

We denote by $\lambda_{1}$ and $\phi_{1}(x)>0$ the principal eigenpair of the problem

$$
u^{\prime \prime}+\lambda a(x) u=0, \text { for } 0<x<1, \quad u(0)=u(1)=0 .
$$

Theorem 4.1. Assume that a(x) satisfies either one of the conditions (4.3) and (4.4), while $g(u)$ satisfies the conditions (4.5) and (4.6). Then the problem (4.2) has a curve of positive solutions bifurcating from zero at $\lambda=\lambda_{1}$. This curve tends to infinity as $\lambda \rightarrow \lambda_{1}$. Moreover, as this curve tends to infinity, the solutions on it tend to a constant multiple of $\phi_{1}(x)$.

Proof. By the standard theory on bifurcation from zero there is a curve of positive solutions bifurcating from zero at $\lambda=\lambda_{1}$, see [3]. It is easy to see that all solutions on this curve stay positive, using uniqueness of solutions for initial value problems. This solution curve continues globally, since at each point either the implicit function theorem or bifurcation theorem of M.G. Crandall and P.H. Rabinowitz [4] applies, see [10], [8] (it is for this step that we need our assumptions on $a(x)$ ). By the global bifurcation theorem of P. Rabinowitz this curve extends to infinity (it cannot go to zero at another eigenvalue, since such solutions are sign changing). As before, we show that on this curve $\lambda$ lies between two positive constants.

Let $\mu=|u|_{L^{\infty}[0,1]}$. As before, we scale $u=\mu v$. The problem (4.2) transforms into

$$
v^{\prime \prime}+\lambda a(x)(v+v g(\mu v))=0, \text { for } 0<x<1, \quad v(0)=v(1)=0 .
$$

Multiplying the equation in (4.7) by $\phi_{1}(x)$, and integrating, we get

$$
\left(\lambda_{1}-\lambda\right) \int_{0}^{1} a(x) v(x) \phi_{1}(x) d x=\lambda \int_{0}^{1} a(x) v(x) g(\mu v(x)) \phi_{1}(x) d x .
$$


We claim that the integral on the left is bounded below by a positive constant as $\mu \rightarrow \infty$. Indeed, from the equation (4.7) we see that $v(x)$ is a concave positive function, with maximum value one, so that clearly it is bounded from below by a positive function (e.g. by $\min (x, 1-x)$ ), uniformly in $\mu$. So once we prove that the integral on the right in (4.8) tends to zero, it will follow that $\lambda \rightarrow \lambda_{1}$ as $\mu \rightarrow \infty$. Define $\bar{G}(v)=\int_{0}^{v} \operatorname{tg}(\mu t) d t$. Clearly, in terms of the functions defined above

$$
\bar{G}(v)=\frac{1}{\mu} v G(\mu v)-\frac{1}{\mu^{2}} H(\mu v)
$$

We now proceed similarly to R. Schaaf and K. Schmitt [13],

$$
\begin{aligned}
\int_{0}^{1} \operatorname{avg}(\mu v) \phi_{1} d x & =\int_{0}^{1} \frac{d}{d x}[\bar{G}(v)-\bar{G}(1)] \frac{a \phi_{1}}{v^{\prime}} d x \\
& =-\int_{0}^{1}[\bar{G}(v)-\bar{G}(1)] \frac{\left(a \phi_{1}\right)^{\prime} v^{\prime}-a \phi_{1} v^{\prime \prime}}{v^{\prime 2}} d x .
\end{aligned}
$$

Using (4.9), we see that by our assumptions $\bar{G}(v) \rightarrow 0$, as $\mu \rightarrow \infty$, uniformly in $v \in[0,1]$. Since $\lambda$ is bounded on the curve, we see from the equation (4.7) that $v^{\prime \prime}$ and $v^{\prime}$ stay bounded uniformly in $\mu$. Hence the integral on the right tends to zero as $\mu \rightarrow \infty$, and so $\lambda \rightarrow \lambda_{1}$. (Observe that the integration by parts is admissible, since near any point of maximum of $v(x)$, call this point $\xi, \bar{G}(v(x))-\bar{G}(1)$ goes to zero proportionally to $v(x)-1$, which goes to zero proportionally to $(x-\xi)^{2}$, and hence the integrand on the right in (4.10) is bounded.)

For the last claim, we rewrite the problem (4.7) as

$$
v^{\prime \prime}+\lambda_{1} a v=\left(\lambda_{1}-\lambda\right) a v-\lambda a v g(\mu v), \quad-1<x<1, \quad v(-1)=v(1)=0 .
$$

Using the modified Green's function $G(x, \xi)$, see e.g. p. 136 in E.C. Young [15], we express

$$
\begin{aligned}
v(x)=\left(\lambda_{1}-\lambda\right) & \int_{-1}^{1} G(x, \xi) a(\xi) v(\xi) d \xi- \\
& \lambda \int_{-1}^{1} G(x, \xi) a(\xi) v(\xi) g(\mu v(\xi)) d \xi+c \phi_{1}(x),
\end{aligned}
$$

where $c$ is some constant. The first term on the right tends to zero, since $\lambda \rightarrow \lambda_{1}$, as $\mu \rightarrow \infty$, while the second one tends to zero by applying the same integration by parts argument, as we just did in (4.10). It follows that $v(x)$ tends to $c \phi_{1}(x)$. 


\section{Bifurcation for balls in $R^{n}$}

We consider positive solutions of a model Dirichlet problem

$$
\Delta u+\lambda(u+u g(u))=0, \quad x \in B, \quad u=0 \text { for } x \in \partial B,
$$

where $B$ is unit ball in $R^{n}, \lambda$ is a positive parameter, and the function $g(u) \in$ $C^{1}\left(\bar{R}_{+}\right)$is assumed to satisfy the conditions (4.5) and (4.6). By the classical theorem of B. Gidas, W.-M. Ni and L. Nirenberg [6] any positive solution of the problem (4.2) is radially symmetric, i.e. $u=u(r)$, and moreover $u^{\prime}(r)<0$ for all $r \in(0,1)$ (here $r=|x|)$. The equation (5.1) then takes the form

$$
u^{\prime \prime}+\frac{n-1}{r}+\lambda(u+u g(u))=0, \quad 0<r<1, \quad u^{\prime}(0)=u(1)=0 .
$$

We denote by $\lambda_{1}$ the principal eigenvalue of $-\Delta$ on $B$ with zero boundary condition, and by $\phi_{1}=\phi_{1}(r)$ the corresponding eigenfunction.

Theorem 5.1. Assuming the conditions (4.5) and (4.6), the problem (5.1) has a curve of positive solutions bifurcating from zero at $\lambda=\lambda_{1}$. This curve tends to infinity as $\lambda \rightarrow \lambda_{1}$. Moreover, as this curve tends to infinity, the solutions on it tend to a constant multiple of $\phi_{1}(x)$.

Proof. The proof is similar to the Theorem 4.1. There is a curve of positive solutions bifurcating from zero at $\lambda=\lambda_{1}$. By [7] this curve continues globally, and by the global bifurcation theorem of P. Rabinowitz, it has to tend to infinity. We show that this happens when $\lambda \rightarrow \lambda_{1}$.

As before, we show that $\lambda$ stays bounded on the curve. (This also follows by the Sturm comparison theorem, applied to (5.2).) With $\mu=u(0)$, the maximum value of solution, we scale as before $u=\mu v$, obtaining

$$
-\Delta v=\lambda(v+v g(\mu v)), \quad x \in B, \quad v=0 \text { for } x \in \partial B,
$$

Since $\lambda$ is bounded, we see that the right hand side is bounded in $L^{p}(B)$ for any $p>1$. This implies a bound on $v$ in $W^{2, p}(B)$. By Sobolev imbedding, and Schauder estimates we conclude a bound on $v$ in $C^{2, \alpha}(B)$. Multiplying the equation in (5.3) by $\phi_{1}$ and integrating, we have in view of radial symmetry

$$
\left(\lambda_{1}-\lambda\right) \int_{0}^{1} v(r) \phi_{1}(r) r^{n-1} d r=\lambda \int_{0}^{1} v(r) g(\mu v(r)) \phi_{1}(r) r^{n-1} d r .
$$


With $\bar{G}$ as defined above, we have

$$
\begin{gathered}
\int_{0}^{1} v g(\mu v) \phi_{1} r^{n-1} d r=\int_{0}^{1} \frac{d}{d r}[\bar{G}(v)-\bar{G}(1)] \frac{\phi_{1} r^{n-1}}{v^{\prime}} d r \\
=-\int_{0}^{1}[\bar{G}(v)-\bar{G}(1)] \frac{\left(\phi_{1} r^{n-1}\right)^{\prime} v^{\prime}-\phi_{1} r^{n-1} v^{\prime \prime}}{v^{\prime 2}} d r .
\end{gathered}
$$

Since $v^{\prime}(r)$ and $v^{\prime \prime}(r)$ are uniformly bounded, we conclude as in the proof of Theorem 4.1, that the integral on the right tends to zero as $\mu \rightarrow \infty$.

We claim that the integral on the left in (5.4) is bounded below by a positive constant. Assuming otherwise, the decreasing function $v(r)$ would have to get small on most of the interval $(0,1)$, i.e. we could find a point $\xi \in(0,1)$ with both $v(\xi)$ and $v^{\prime}(\xi)$ small. Now look at the ODE version of the equation (5.3). This equation admits a trivial solution, and the term $\lambda(v+v g(\mu v))$ is bounded. By continuous dependence on initial data, $v(r)$ has to be close to the trivial solution, and then $v(0)$ has to be small, contradicting $v(0)=1$. This proves the claim, and it follows that $\lambda \rightarrow \lambda_{1}$, as $\mu \rightarrow \infty$.

Turning to the last claim, we rewrite the problem (5.3) as

$$
\Delta v+\lambda_{1} v=h(v) \equiv\left(\lambda_{1}-\lambda\right) v-\lambda v g(\mu v), \quad x \in B, \quad v=0 \text { for } x \in \partial B,
$$

Let $\bar{H}=\left\{u \in H_{0}^{1}(B) \mid \int_{B} u \phi_{1} d x=0\right\}$. Observe that $h(v)$ tends to zero weakly in $L^{2}(B)$ (as $\lambda \rightarrow \lambda_{1}$ the first term goes to zero, while the second term in $h(v)$ goes weakly to zero by an argument similar to (5.5)). Since the problem (5.6) is solvable, $h(v) \in \bar{H}$. We now write the solution of (5.6) as

$$
v=\left(\Delta+\lambda_{1} I\right)^{-1} h(v)+c \phi_{1} .
$$

Since the operator $\left(\Delta+\lambda_{1} I\right)^{-1}$ is compact on $\bar{H}$, we see (after a boot-strap) that the first term on the right goes uniformly to zero, and hence $v(x) \rightarrow c \phi_{1}$, as $\lambda \rightarrow \lambda_{1}$.

Remark Proceeding as in D. Costa et al [2], one can prove a similar result for general domains. However, in that case one has to work with a continuum of solution bifurcating from zero, rather than a curve of solutions.

\section{References}

[1] A. Canãda, Multiplicity results near the principal eigenvalue for boundaryvalue problems with periodic nonlinearity, Math. Nachr. 280(3) (2007), $235-241$.

[2] D. Costa, H. Jeggle, R Schaaf and K. Schmitt, Oscillatory perturbations of linear problems at resonance, Results Math. 14(3-4) (1988), 275-287. 
[3] M. G. Crandall and P. H. Rabinowitz, Bifurcation from simple eigenvalues, Journal of Functional Analysis 8 (1971), 321-340.

[4] M. G. Crandall and P. H. Rabinowitz, Bifurcation, perturbation of simple eigenvalues and linearized stability, Arch. Rational Mech. Anal. 52 (1973), $161-180$.

[5] A. Galstyan, P. Korman and Y. Li, On the oscillations of the solution curve for a class of semilinear equations, J. Math. Anal. Applic. 321(2), (2006), $576-588$.

[6] B. Gidas, W.-M. Ni and L. Nirenberg, Symmetry and related properties via the maximum principle, Commun. Math. Phys. 68 (1979), 209-243.

[7] P. Korman, Solution curves for semilinear equations on a ball, Proc. Amer. Math. Soc. 125(7) (1997), 1997-2005.

[8] P. Korman, Global solution branches and exact multiplicity of solutions for two point boundary value problems, Handbook of Differential Equations, Ordinary Differential Equations, Vol. 3, Edited by A. Canãda, P. Drabek and A. Fonda, Elsevier Science, North Holland, (2006), 547-606.

[9] P. Korman and Y. Li, Infinitely many solutions at a resonance, Electron. J. Diff. Eqns., Conf. 05, pp. (2000), 105-111.

[10] P. Korman and T. Ouyang, Solution curves for two classes of boundary-value problems, Nonlinear Anal. 27(9) (1996), 1031-1047.

[11] E. M. Landesman and A. C. Lazer, Nonlinear perturbations of linear elliptic boundary value problems at resonance, J. Math. Mech. 19 (1970), 609-623.

[12] Y. V. Sidorov, M. V. Fedoryuk and M. I. Shabunin, Lectures on the Theory of Functions of Complex Variable, Mir Publishers, Moscow (1985).

[13] R. Schaaf and K. Schmitt, A class of nonlinear Sturm-Liouville problems with infinitely many solutions, Trans. Amer. Math. Soc. 306(2) (1988), 853-859.

[14] S.-H. Wang, On the evolution and qualitative behaviors of bifurcation curves for a boundary value problem, Nonlinear Anal. TMA 67(5) (2007), $1316-1328$

[15] E. C. Young, Partial Differential Equations, Allyn and Bacon, Inc. Boston (1972).

Philip Korman

Department of Mathematical Sciences

University of Cincinnati

Cincinnati Ohio 45221-0025

USA

e-mail: kormanp@math.uc.edu

Received: 4 April 2007

Accepted: 23 October 2007

Published Online First 03 October 2008 
